\title{
Near-Capacity Iteratively Decoded Binary Self-Concatenated Code Design Using EXIT Charts
}

\author{
Muhammad Fasih Uddin Butt ${ }^{1,2}$, Raja Ali Riaz ${ }^{1,2}$, Soon Xin Ng${ }^{1}$ and Lajos Hanzo ${ }^{1}$ \\ ${ }^{1}$ School of ECS, University of Southampton, SO17 1BJ, United Kingdom. \\ Email: \{mfub06r, rar06r, sxn, lh\}@ecs.soton.ac.uk, http://www-mobile.ecs.soton.ac.uk \\ ${ }^{2}$ Dept of EE, COMSATS Institute of Information Technology, Islamabad, 44000, Pakistan, http://ciit.edu.pk
}

\begin{abstract}
In this treatise Extrinsic Information Transfer (EXIT) charts are used to design binary Self-Concatenated Convolutional Codes employing Iterative Decoding (SECCC-ID) for communicating over both uncorrelated Rayleigh fading and Additive White Gaussian Noise (AWGN) channels. Recursive Systematic Convolutional (RSC) codes are selected as constituent codes, an interleaver is used for randomising the extrinsic information exchange of the constituent codes, while a puncturer assists us in increasing the achievable bandwidth efficiency. At the receiver, self-iterative decoding is invoked for exchanging extrinsic information between the hypothetical decoder components. The convergence behaviour of the decoder is analysed with the aid of bit-based EXIT charts. Finally, we propose an attractive system configuration, which is capable of operating within about $1 \mathrm{~dB}$ of the information-theoretic limits.
\end{abstract}

\section{INTRODUCTION}

The philosophy of Concatenated coding schemes was proposed by Forney in [1]. Turbo codes constitute a class of error correction codes (ECC) based on parallel concatenated convolutional codes (PCCC) of two or more constituent codes which were developed in [2]. They are high-performance codes capable of operating near the Shannon limit [3]. Since their invention they have found diverse applications in bandwidthlimited communication systems, where the maximum achievable information rate has to be supported in the presence of transmission errors due to Additive White Gaussian Noise (AWGN) and channel fading. Various turbo trellis coded modulation (TTCM) schemes were proposed in [4], [5] and [6]. Serially concatenated convolutional codes (SCCC) [7] have been shown to yield a performance comparable, and in some cases superior, to turbo codes. Iteratively-Decoded SelfConcatenated Convolutional Codes (SECCC-ID) proposed by Benedetto et al. [8] and Loeliger [9] constitute another attractive family of iterative detection aided schemes.

The concept of Extrinsic Information Transfer (EXIT) charts was proposed by Ten Brink [10] as a tool designed for analysing the convergence behaviour of iteratively decoded systems. EXIT charts constitute a semi-analytical tool used to predict the SNR value where an infinitesimally low Bit Error Ratio (BER) can be achieved without performing timeconsuming bit-by-bit decoding employing a high number of decoding iterations.

SECCC is a low-complexity scheme involving only a single encoder and a single decoder. An EXIT chart based analysis of the iterative decoder provides an insight into its decoding

The financial support of COMSATS Institute of Information Technology, Islamabad under the auspices of Higher Education Commission, Pakistan and that of the EPSRC UK, as well as of the EU Optimix project is gratefully acknowledged. convergence behaviour and hence it is helpful for finding the best coding schemes for SECCCs.

An SECCC-ID scheme was designed using Trellis Coded Modulation (TCM) as constituent codes with the aid of EXIT charts in [11]. The proposed design in [11] was symbol-based, therefore it had the inherent problem of exhibiting a mismatch between the EXIT curve and the bit-by-bit decoding trajectory. The main reason for the mismatch was that the EXIT charts were generated based on the assumption that the extrinsic information and the systematic information part of each TCM encoded symbol are independent of each other, which had a limited validity, since both the systematic and the parity bits were being transmitted together as a single $2^{n+1}$-ary symbol. More explicitly, the coded bits in each coded symbol are correlated [12-14]. Nonetheless, we found that the EXIT charts of the symbol-based SECCC scheme can be beneficially used as upper bounds, since the actual EXIT chart tunnel is always wider than the predicted EXIT chart tunnel. Hence, the analysis was still valid, since it assisted us in finding the convergence threshold.

In this paper, we eliminate the mismatch inherited by the symbol-based design by proposing a bit-based SECCC-ID design in order to create flexible SECCC schemes capable of efficiently operating over both AWGN and uncorrelated Rayleigh fading channels. EXIT charts have been used to find the convergence behaviour of these schemes. Some of the proposed SECCC schemes perform within about $1 \mathrm{~dB}$ from the $A W G N$ and Rayleigh fading channels' capacity.

The organisation of the paper is as followed. After presenting our system model in Section II, we discuss our code design procedure using EXIT charts in Section III. Our results are discussed in Section IV and our conclusions are offered in Section V.

\section{SySTEM Model}

We consider a rate $R=1 / 2$ SECCC scheme as an example to highlight the various system concepts considered in this paper. In all the examples we use Gray-coded Quadrature Phase-Shift Keying (QPSK) modulation and both the Additive White Gaussian Noise (AWGN) as well as uncorrelated Rayleigh fading channels are considered.

As shown in Fig. 1, the input bit sequence $\left\{b_{1}\right\}$ of the selfconcatenated encoder is interleaved for yielding the bit sequence $\left\{b_{2}\right\}$. The resultant bit sequences are parallel-to-serial converted and then fed to the RSC encoder with generator polynomials $\left(G_{0}=13, G_{1}=15, G_{2}=17\right)$ in octal format having rate $R_{1}=\frac{1}{3}$ and memory $\nu=3$. Hence for every bit input to the SECCC encoder there are six output bits of 


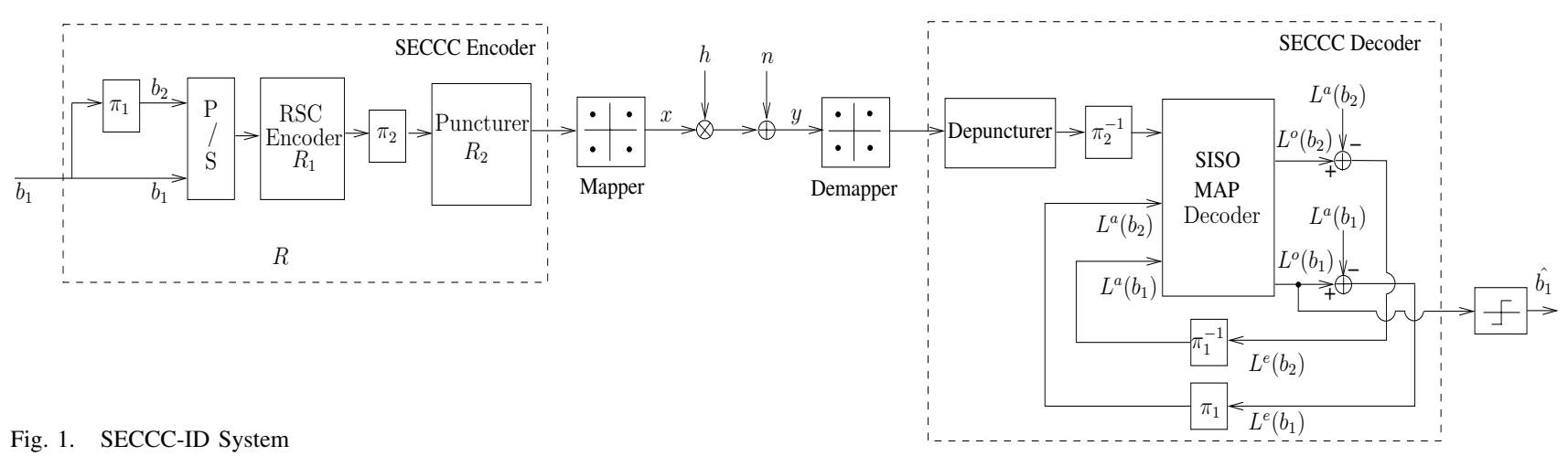

the RSC encoder. At the output of the encoder there is an interleaver and then a rate $R_{2}=\frac{1}{3}$ puncturer, which punctures (does not transmit) two bits out of three encoded bits. Hence, the overall code rate, $\mathrm{R}$ can be derived based on [15] as:

$$
R=\frac{R_{1}}{2 \times R_{2}}=\frac{1}{2}\left(\frac{1}{3\left(\frac{1}{3}\right)}\right)=\frac{1}{2},
$$

Therefore, at the output of the puncturer the number of encoded bits reduces from six to two bits, namely $\left(c_{0} c_{1}\right)$. Puncturing is used in order to increase the achievable bandwidth efficiency $\eta$. It can be observed that different codes can be designed by changing $R_{1}$ and $R_{2}$. These bits are then mapped to a QPSK symbol as $x=\mu\left(c_{0} c_{1}\right)$, where $\mu($.$) is the$ Gray-coded mapping function. Hence the bandwidth efficiency is given by $\eta=1 \mathrm{bit} / \mathrm{s} / \mathrm{Hz}$, which is for a Nyquist roll-offfactor of $\alpha=0$. The QPSK symbol $x$ is then transmitted over the communication channel. At the receiver side the received symbol is given by: $y=h x+n$, where $h$ is the channel's non-dispersive fading coefficient and $n$ is the AWGN having a variance of $\frac{N_{0}}{2}$ per dimension. This signal is then used by a soft demapper for calculating the conditional probability density function (PDF) of receiving $y$, when $x^{(m)}$ was transmitted:

$$
P\left(y \mid x^{(m)}\right)=\frac{1}{\pi N_{0}} \exp \left(-\frac{\left|y-h x^{(m)}\right|^{2}}{N_{0}}\right),
$$

where $x^{(m)}=\mu\left(c_{0} c_{1}\right)$ is the hypothetically transmitted QPSK symbol for $m \in\{0,1,2,3\}$. Then these PDFs are passed to a soft depuncturer, which converts the PDFs to bit-based Log-Likelihood Ratios (LLRs) and inserts zero LLRs at the punctured bit positions. These LLRs are then deinterleaved and fed to the Soft-Input Soft-Output (SISO) Maximum A Posteriori Probability (MAP) decoder [16]. The decoder is a self-concatenated decoder. It first calculates the extrinsic LLR of the information bits, namely $L_{e}\left(b_{1}\right)$ and $L_{e}\left(b_{2}\right)$. Then they are appropriately interleaved to yield the a priori LLRs of the information bits, namely $L_{a}\left(b_{1}\right)$ and $L_{a}\left(b_{2}\right)$, as shown in Fig. 1. Self-concatenated decoding proceeds, until a fixed number of iterations is reached.

\section{Code Design Procedure Using EXIT Charts}

EXIT charts constitute a powerful tool designed for analysing the convergence behaviour of concatenated codes without time-consuming bit-by-bit simulation of the actual system. They analyse the input/output mutual information characteristics of a SISO decoder by modelling the a priori LLRs and computing the corresponding mutual information between the extrinsic LLRs and the corresponding bitdecisions. However, the employment of EXIT charts assumes having a sufficiently high interleaver length, so that the extrinsic LLRs can be rendered independent Gaussian distributed. The SNR value, where the turbo-cliff [2] in the BER curve of a concatenated code appears can be successfully predicted with the aid of EXIT charts.

The decoding model of the SECCC-ID scheme can be represented by Fig. 2. The information bit sequence is $\underline{U}$, which is encoded, yielding the coded symbol sequence $\underline{X}$, which is then transmitted over the communication channel. The received symbol sequence is given by $\underline{Y}$, which is then fed to the SISO SECCC decoder. The a priori channel models the a priori probabilities of the information bit sequence $\underline{U}$ by $\underline{A}(U)$ and its interleaved version $\underline{W}$ by $\underline{A}(W)$. The SECCC SISO decoder then computes both the a posteriori bit probabilities $\underline{O}(U)$ and the extrinsic bit probabilities $\underline{E}(U)$ and $\underline{E}(W)$. An EXIT chart plots the extrinsic information $I_{E}$ as a function of the a priori information $I_{A}$. In the context of SECCC, $I_{A}$ is the joint a priori information of $U$ and $W$, and $I_{E}$ is the joint extrinsic information of $U$ and $W$.

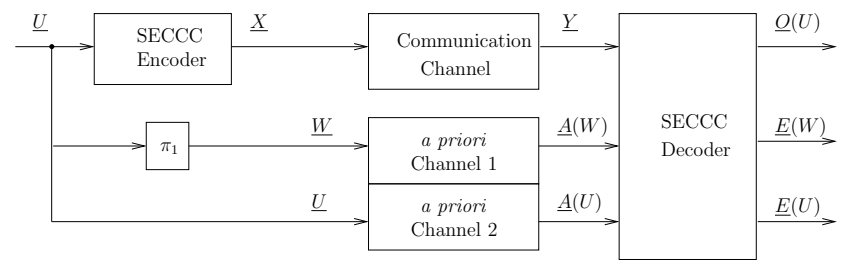

Fig. 2. Decoding model for an SECCC-ID scheme [11].

The EXIT charts of self-concatenated codes are typically similar to those of the parallel concatenated TTCM schemes $[13,14]$, where an open EXIT tunnel exists if the EXIT curve does not intersect with the straight line connecting the point $\left(I_{A}=0, I_{E}=0\right)$ to the point $\left(I_{A}=1, I_{E}=1\right)$ in the EXIT chart. In [11] EXIT charts were successfully used to compare the performance of non-binary SECCC schemes by employing the same method. The various coding schemes considered in this paper are characterised in Table I. They are identified by the code rate $\left(R_{1}\right)$, puncturing rate $\left(R_{2}\right)$, overall code rate $(R)$, code memory $\nu$ and bandwidth efficiency, expressed in bit/s/Hz, as $\eta$. The $E_{b} / N_{0}$ decoding convergence threshold, beyond which the EXIT tunnel becomes 'just' open is denoted by $T h$., although this does not necessarily imply that the $\left(I_{A}, I_{E}\right)=(1,1)$ point of 'perfect convergence' can be reached because some of the decoding trajectories are curtailed owing to the limited interleaver length used. This is why the slightly different term, tunnel $T l$. was introduced, which specifies the $E_{b} / N_{0}$ value where there is a more widely open 
EXIT tunnel leading to the $(1,1)$ point and where decoding convergence to an infinitesimally low BER value can always be achieved, provided that the interleaver length is large and the number of iterations is sufficiently high [10]. Furthermore, the channel capacity limit $\omega$ is also expressed in $\mathrm{dBs}$ [17], as tabulated in Table I. For $R_{1}=1 / 2$ and $\nu=2$, the generator polynomial $G=(7,5)$ is used, whereas for $\nu=3, G=$ $(13,15)$ is employed. For $R_{1}=1 / 3$ and $\nu=3, G=(13,15,17)$ is used.

\begin{tabular}{|c|c|c|c|c|c|c|c|c|}
\hline \multirow[t]{2}{*}{$\begin{array}{l}\text { SECCC-ID } \\
\text { Scheme }\end{array}$} & \multirow[t]{2}{*}{$\nu$} & \multirow{2}{*}{$\begin{array}{l}\eta_{\text {(bit/s }} \\
/ \mathrm{Hz} \text { ) }\end{array}$} & \multicolumn{3}{|c|}{$\begin{array}{l}\text { AWGN Channel } \\
E_{b} / N_{0}(\mathrm{~dB})\end{array}$} & \multicolumn{3}{|c|}{$\begin{array}{l}\text { Rayleigh Channel } \\
E_{b} / N_{0}(\mathrm{~dB})\end{array}$} \\
\hline & & & $T h$. & $T l$. & $\omega$ & $T h$. & $T l$. & $\omega$ \\
\hline $\mathrm{R}_{1}=1 / 2, \mathrm{R}_{2}=3 / 4$, & 2 & 0.67 & 1.4 & 1.6 & -0.6 & 2.6 & 2.8 & 1 \\
\hline $\mathrm{R}=1 / 3$ & 3 & 0.67 & 0.7 & 0.8 & -0.6 & 1.85 & 1.95 & 1 \\
\hline $\mathrm{R}_{1}=1 / 2, \mathrm{R}_{2}=1 / 2$ & 2 & 1 & 2.3 & 2.4 & 0.19 & 4.6 & 4.75 & 1.83 \\
\hline $\mathrm{R}=1 / 2$ & 3 & 1 & 1.55 & 1.6 & 0.19 & 3.6 & 3.7 & 1.83 \\
\hline $\mathrm{R}_{1}=1 / 2, \mathrm{R}_{2}=1 / 3$ & 2 & 1.5 & 5.4 & 5.6 & 2 & 13.5 & 13.7 & 6 \\
\hline $\mathrm{R}=3 / 4$ & 3 & 1.5 & 3.8 & 4.0 & 2 & 9.3 & 9.5 & 6 \\
\hline $\begin{array}{l}\mathrm{R}_{1}=1 / 3, \mathrm{R}_{2}=2 / 3, \\
\mathrm{R}=1 / 4\end{array}$ & 3 & 0.5 & 0.4 & 0.5 & -0.8 & 1.2 & 1.25 & -0.2 \\
\hline $\begin{array}{l}\mathrm{R}_{1}=1 / 3, \mathrm{R}_{2}=1 / 3, \\
\mathrm{R}=1 / 2\end{array}$ & 3 & 1 & 1.5 & 1.6 & 0.19 & 3.6 & 3.7 & 1.83 \\
\hline $\begin{array}{l}\mathrm{R}_{1}=1 / 3, \mathrm{R}_{2}=1 / 4, \\
\mathrm{R}=2 / 3\end{array}$ & 3 & 1.33 & 2.7 & 2.8 & 1.5 & 6.5 & 6.7 & 3 \\
\hline
\end{tabular}

TABLE I

VARIOUS SECCC-ID SCHEMES AND THEIR DECODING CONVERGENCE THRESHOLDS AND EXIT TUNNELS.

The EXIT charts recorded for the binary SECCC-ID schemes of Table I are shown in Fig. 3, 4, 5 and 6. The two EXIT curves represent the two hypothetical decoder components of the SECCC scheme, while the stair-case-shaped trajectories correspond to iterating between them. Since these are identical components, we only have to compute the EXIT curve of one component and the other is its mirror image with respect to the diagonal line. The EXIT curves of the hypothetical decoder components are plotted within the same EXIT chart together with their corresponding decoding trajectory for the sake of visualizing the transfer of extrinsic information between the decoders. The EXIT curves of the proposed scheme exactly match the decoding trajectories computed from the bit-by-bit simulations.

The EXIT curves and the two distinct decoding trajectories were recorded for the best-performing binary SECCC schemes operating closest to the Rayleigh channel's capacity which, are given in Fig. 3 and 4. These were recorded by using $10^{3}$ transmission frames, each consisting of $24 \times 10^{3}$ information bits for calculating the EXIT curve, and $10^{3}$ frames each consisting of $120 \times 10^{3}$ information bits for calculating the decoding trajectories.

In Fig. 3, the scheme using $R_{1}=1 / 2, R_{2}=3 / 4$, $\nu=3$ acquires an open EXIT tunnel at $E_{b} / N_{0}=1.95 \mathrm{~dB}$, when communicating over an uncorrelated Rayleigh fading channel. For this scheme the threshold is achieved at $1.85 \mathrm{~dB}$ according to Table I, which is $0.85 \mathrm{~dB}$ away from capacity. The SECCC scheme employing $R_{1}=1 / 3, R_{2}=2 / 3, \nu=3$ starts to exhibit an open tunnel at $E_{b} / N_{0}=1.25 \mathrm{~dB}$, when communicating over uncorrelated Rayleigh fading channels, as shown in Fig. 4. For this scheme the threshold is achieved at $1.2 \mathrm{~dB}$ according to Table $\mathrm{I}$, which is $1.4 \mathrm{~dB}$ away from capacity.

The EXIT curves along with their corresponding decoding trajectory 'snap-shots' recorded for the case of AWGN chan-

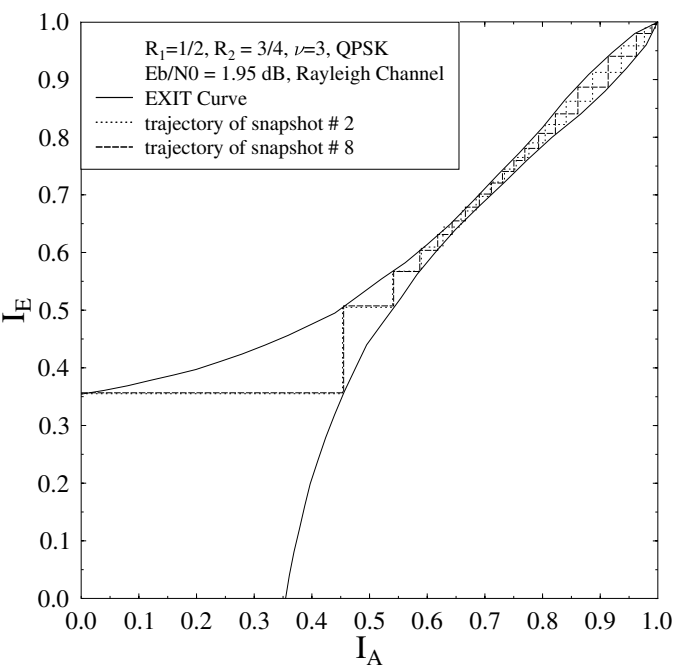

Fig. 3. EXIT chart and two 'snap-shot' decoding trajectories for $R_{1}=1 / 2$ and $R_{2}=3 / 4$, QPSK-assisted SECCC-ID, $\nu=3, \eta=0.67 \mathrm{bit} / \mathrm{s} / \mathrm{Hz}$ at $E_{b} / N_{0}=$ $.95 \mathrm{~dB}$, for transmission over a Rayleigh fading channel.

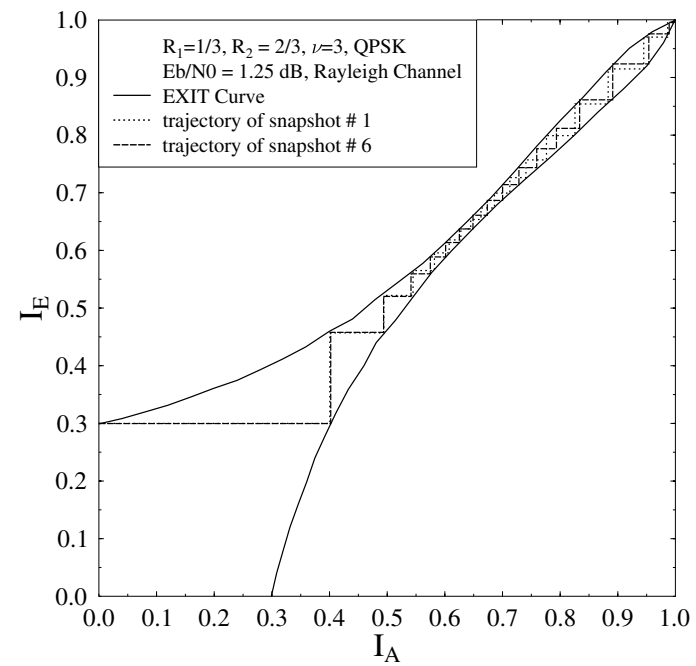

Fig. 4. EXIT chart and two 'snap-shot' decoding trajectories for $R_{1}=1 / 3$ and $R_{2}=2 / 3$, QPSK-assisted SECCC-ID, $\nu=3, \eta=0.5 \mathrm{bit} / \mathrm{s} / \mathrm{Hz}$ at $E_{b} / N_{0}=$ $1.25 \mathrm{~dB}$, for transmission over a Rayleigh fading channel.

nels are shown in Fig. 5 and 6. Fig. 5 is exemplifying the achievable performance of a $\nu=2$ RSC code. We found that when employing the $\nu=2$ RSC code, all SECCC schemes exhibited EXIT curves having similar trends as in Fig. 5, where the tunnel at the top-right corner becomes very narrow. Hence, a higher SNR was required for the decoding trajectory to pass through the tunnel. As a result, their performance tends to be farther away from the channel capacity. For example, for $\nu=2, R_{1}=1 / 2$ and $R_{2}=1 / 2$, an open EXIT tunnel emerges at $E_{b} / N_{0}=2.40 \mathrm{~dB}$. The threshold is shown in Table I to be at $E_{b} / N_{0}=2.30 \mathrm{~dB}$, which is $2.11 \mathrm{~dB}$ away from capacity. By contrast, when employing $\nu=3, R_{1}=1 / 2$ and $R_{2}=3 / 4$, an open EXIT tunnel emerges at $0.8 \mathrm{~dB}$ as shown in Fig. 6, and the threshold is at $E_{b} / N_{0}=0.7 \mathrm{~dB}$, as shown in Table I, which is $1.3 \mathrm{~dB}$ away from capacity.

The employment of the interleaver, $\pi_{1}$ seen in Fig. 1 and used in all of the schemes considered in Table I renders the information bits, more-or-less uncorrelated. This is a necessary requirement for the employment of EXIT charts, because they require the LLRs of the information bits to be Gaussian distributed. The interleaver used after the RSC encoder of 


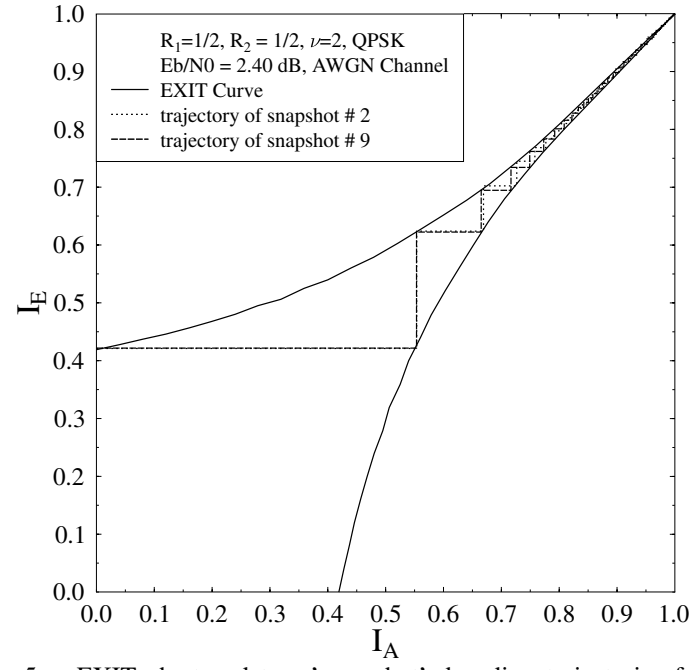

Fig. 5. EXIT chart and two 'snap-shot' decoding trajectories for $R_{1}=1 / 2$ and $R_{2}=1 / 2$, QPSK-assisted SECCC-ID, $\nu=2, \eta=1 \mathrm{bit} / \mathrm{s} / \mathrm{Hz}$ at $E_{b} / N_{0}=$ $2.40 \mathrm{~dB}$, for transmission over an AWGN channel.

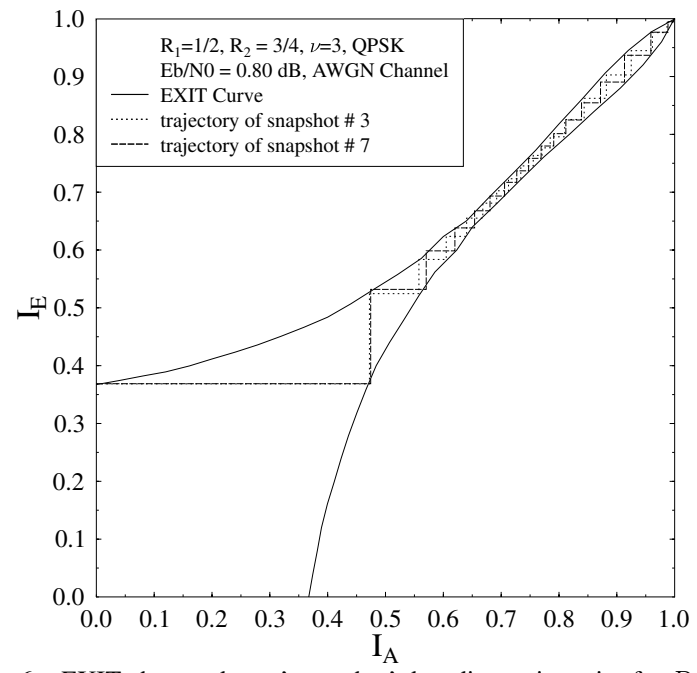

Fig. 6. EXIT chart and two 'snap-shot' decoding trajectories for $R_{1}=1 / 2$ and $R_{2}=3 / 4$, QPSK-assisted SECCC-ID, $\nu=3, \eta=0.67 \mathrm{bit} / \mathrm{s} / \mathrm{Hz}$ at $E_{b} / N_{0}=$ $0.80 \mathrm{~dB}$, for transmission over an AWGN channel.

Fig. 1, namely $\pi_{2}$, randomises the coded bits before the puncturer.

\section{RESUltS AND Discussions}

The EXIT charts discussed in Section III were used to find the best SECCC schemes for $\nu=\{2,3\}$, when communicating over AWGN and uncorrelated Rayleigh fading channels.

The convergence threshold predicted by the EXIT chart analysis detailed in Section III, closely matches with the actual convergence threshold observed in the BER curve given by the specified $E_{b} / N_{0}$ value, where there is a sudden drop of the BER after a certain number of decoding iterations, as shown in Figs. 7, 8 and 9. Hence it becomes possible to attain an infinitesimally low BER beyond the convergence threshold, provided that the block length is sufficiently long and the number of decoding iterations is sufficiently high. Again, the BER versus $E_{b} / N_{0}$ performance curves of the various QPSK-assisted SECCC-ID schemes recorded from our bit-bybit simulations are shown in Figs. 7, 8 and 9. As mentioned, we considered an information block length of $120 \times 10^{3}$ bits per frame, for $10^{3}$ frames and the number of decoding iterations
(I) varied from 50 to 80 . Figs. 7,8 and 9 show the $E_{b} / N_{0}$ difference between the channel capacity and the convergence threshold for the best SECCC-ID schemes at a given code memory $\nu$.

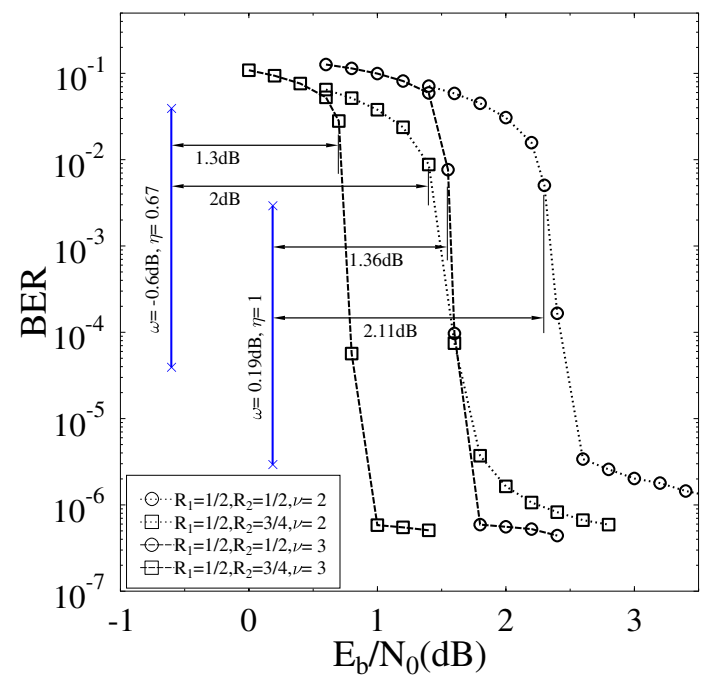

Fig. 7. The BER versus $E_{b} / N_{0}$ performance of various QPSK-assisted SECCC-ID schemes, $R_{1}=1 / 2$ and $I=80$ decoding iterations for $\nu=2$ and $I=50$ decoding iterations for $\nu=3$, operating over AWGN channel.

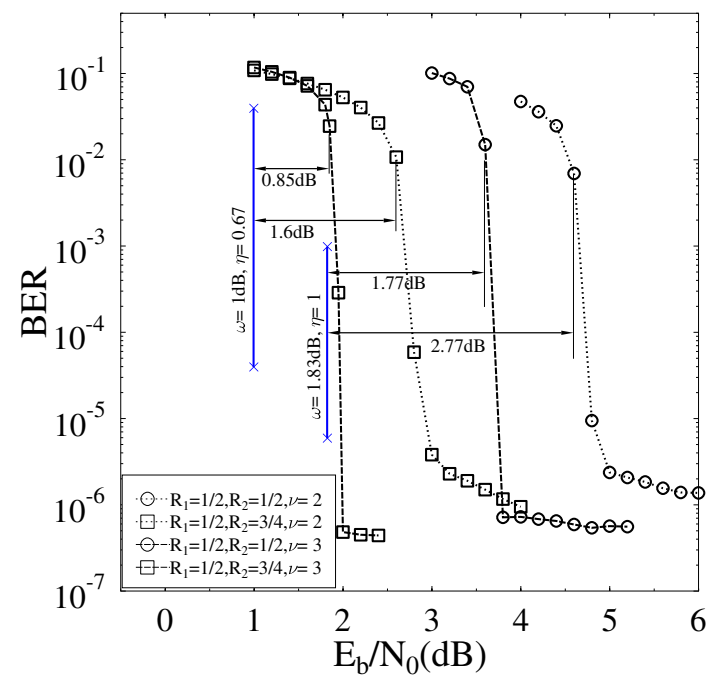

Fig. 8. The BER versus $E_{b} / N_{0}$ performance of various QPSK-assisted SECCC-ID schemes, $R_{1}=1 / 2$ and $I=80$ decoding iterations for $\nu=2$ and $I=50$ decoding iterations for $\nu=3$, operating over uncorrelated Rayleigh fading channel.

It can be observed from Fig. 7 that by increasing the code memory $\nu$ from 2 to 3 , in the case of $R_{1}=1 / 2$ and a higher puncturing rate of $R_{2}=3 / 4$ there is a $0.7 \mathrm{~dB}$ gain and $0.75 \mathrm{~dB}$ gain when communicating over AWGN and uncorrelated Rayleigh fading channel, respectively. Similarly, observe from Fig. 7, that upon increasing $\nu$ from 2 to 3, in the case of $R_{1}=1 / 2$ and $R_{2}=1 / 2$, there is a $0.75 \mathrm{~dB}$ gain and $1 \mathrm{~dB}$ gain when communicating over AWGN and uncorrelated Rayleigh fading channel, respectively. Furthermore, as seen from Figs. 7 and 8 , the scheme using $R_{1}=1 / 2, R_{2}=1 / 2$ and $\nu=3$ is operating within $1.36 \mathrm{~dB}$ and within $1.77 \mathrm{~dB}$ from the capacity in case of AWGN and Rayleigh fading channels, respectively. As observed from Table I, in the case of a lower puncturing rate of $R_{2}=1 / 3$ and $R_{1}=1 / 2$, the $\nu=3$ code performs better compared to the $\nu=2$ code. 
Under AWGN channel conditions the gain achieved is $1.6 \mathrm{~dB}$, whereas under Rayleigh fading channel conditions it is $4.2 \mathrm{~dB}$, but even this higher gain is insufficient to approach capacity. For the scheme considered the difference from capacity for $\nu=3$ code, is $1.8 \mathrm{~dB}$ and $3.3 \mathrm{~dB}$ in case of AWGN and Rayleigh fading channels, respectively. This is due to the fact that the puncturing rate is low. The same trends were observed in other cases of lower puncturing rates such as for example, $R_{2}=1 / 3$ and $1 / 4$ with $R_{1}=1 / 3$.

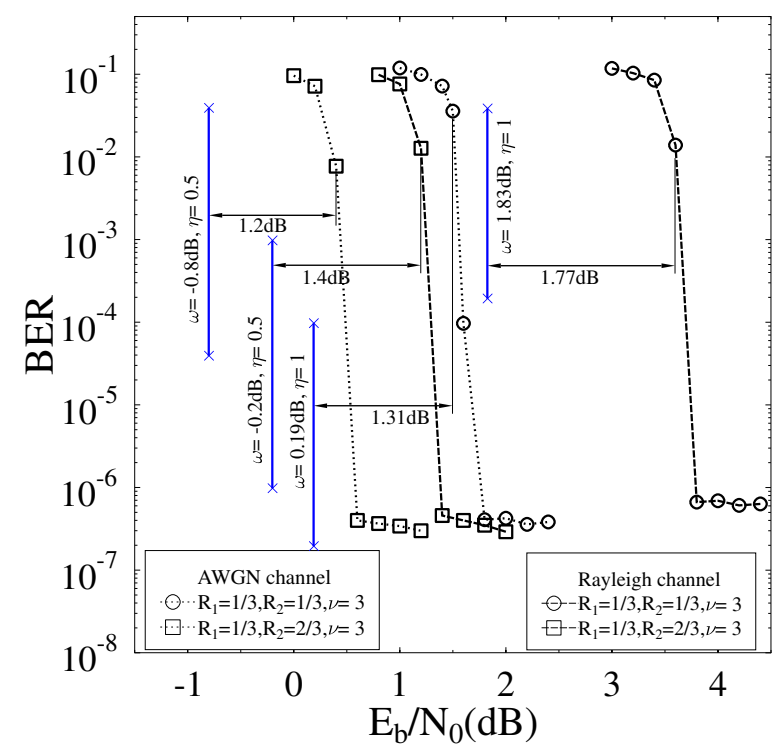

Fig. 9. The BER versus $E_{b} / N_{0}$ performance of various $\nu=3$ QPSKassisted SECCC-ID schemes, $R_{1}=1 / 3$ and $I=50$ decoding iterations, operating over both AWGN and uncorrelated Rayleigh fading channels.

As we can see by studying Table I and Figs. 7 to 9, the actual BER convergence threshold is exactly the same as the convergence threshold predicted by the EXIT charts. Hence, the binary EXIT chart is useful for finding the best SECCCID schemes for having a decoding convergence at the lowest possible $E_{b} / N_{0}$ value.

The best-performing SECCC schemes shown in Figs. 7 and 8 and found from the EXIT chart based design approach are summarised in Table I, which are capable of operating within about $1 \mathrm{~dB}$ the AWGN channel's capacity. For the scheme employing $\nu=3, R_{1}=1 / 2$ and $R_{2}=3 / 4$, the distance from capacity is $1.3 \mathrm{~dB}$ and $0.85 \mathrm{~dB}$ in case of AWGN and Rayleigh fading channels, respectively. For a bandwidth efficiency of $0.67 \mathrm{bit} / \mathrm{s} / \mathrm{Hz}$, the capacity of this scheme [17] is $-0.6 \mathrm{~dB}$ and $1.0 \mathrm{~dB}$ for the QPSK-based discrete-input AWGN and Rayleigh fading channels, respectively. Another scheme, which performs close to capacity is employing $\nu=3$, $R_{1}=1 / 3$ and $R_{2}=2 / 3$, as shown in Fig. 9 and Table I. The difference from capacity for this scheme is $1.2 \mathrm{~dB}$ and $1.4 \mathrm{~dB}$ in case of AWGN and Rayleigh fading channels, respectively.

\section{Conclusions}

We have designed near-capacity SECCC-ID schemes based on their decoding convergence analysis. The SECCC schemes invoke binary RSC codes and different puncturing rates. The puncturer is used to increase the achievable bandwidth efficiency. The interleaver placed before the puncturer helps randomise the puncturing pattern. Good SECCC parameters were found for assisting the SECCC-ID scheme in attaining decoding convergence at the lowest possible $E_{b} / N_{0}$ value, when communicating over both AWGN and uncorrelated Rayleigh fading channels. The SECCC-ID schemes designed are capable of operating within about $1 \mathrm{~dB}$ from the AWGN as well as Rayleigh fading channel's capacity. Our future work will focus on designing SECCC schemes operating closer to capacity, while maintaining a high bandwidth efficiency. In [18] it was suggested that a symbol-based scheme always has a lower convergence threshold compared to an equivalent binary scheme. In order to recover the information loss due to employing binary rather than non-binary schemes, soft decision feedback is required between the SISO MAP decoder and the soft demapper [19]. Furthermore, we will investigate the performance of such SECCC-ID schemes in non-coherently detected cooperative communication systems.

\section{REFERENCES}

[1] G. Forney, Concatenated Codes. MIT Press, Cambridge, MA,, 1966.

[2] C. Berrou, A. Glavieux, and P. Thitimajshima, "Near Shannon limit error correcting coding and decoding: Turbo codes," IEEE Transactions on Communications, pp. 1064-1070, 1993.

[3] C. Shannon, "A mathematical theory of communication," Bell Syst. Tech. Journal, vol. 27, pp. 623-656, 1948.

[4] S. Le Goff, A. Glavieux, and C. Berrou, "Turbo-codes and high spectral efficiency modulation," in IEEE International Conference on Communications, (New Orleans, LA, USA), pp. 645-649, May 1994.

[5] S. Benedetto, D. Divsalar, G. Montorsi, and F. Pollara, "Bandwidth efficient parallel concatenated coding schemes," Electronics Letters, vol. 31, pp. 2067-2069, Nov. 1995.

[6] P. Robertson and T. Worz, "Coded modulation scheme employing turbo codes," Electronics Letters, vol. 31, pp. 1546-1547, Aug. 1995.

[7] S. Benedetto, D. Divsalar, G. Montorsi, and F. Pollara, "Serial concatenated trellis coded modulation with iterative decoding," in IEEE International Symposium on Information Theory, (Ulm), p. 8, June/July 1997.

[8] S. Benedetto, D. Divsalar, G. Montorsi, and F. Pollara, "Selfconcatenated trellis coded modulation with self-iterative decoding," in IEEE Global Telecommunications Conference, vol. 1, (Sydney, NSW, Australia), pp. 585-591, 1998.

[9] H. A. Loeliger, "New turbo-like codes," in IEEE International Symposium on Information Theory, (Ulm), p. 109, June/July 1997.

[10] S. ten Brink, "Convergence behavior of iteratively decoded parallel concatenated codes," IEEE Transactions on Communications, vol. 49, pp. 1727-1737, Oct. 2001.

[11] M. F. U. Butt, S. X. Ng, and L. Hanzo, "Exit chart aided design of near-capacity self-concatenated trellis coded modulation using iterative decoding," in 67th IEEE Vehicular Technology Conference. VTC-'08 Spring., (Marina Bay, Singapore), May 2008.

[12] A. Grant, "Convergence of non-binary iterative decoding," in IEEE Global Telecommunications Conference, vol. 2, (San Antonio, TX, USA), pp. 1058-1062, Nov. 2001.

[13] H. Chen and A. Haimovich, "EXIT charts for turbo trellis-coded modulation," IEEE Communications Letters, vol. 8, pp. 668-670, Nov. 2004.

[14] J. Kliewer, S. X. Ng, and L. Hanzo, "Efficient computation of EXIT functions for nonbinary iterative decoding," IEEE Transactions on Communications, vol. 54, pp. 2133-2136, Dec. 2006.

[15] J. Hagenauer, "Rate-compatible punctured convolutional codes (RCPC codes) and their applications," IEEE Transactions on Communications, vol. 36, pp. 389-400, Apr. 1988.

[16] S. Benedetto, D. Divsalar, G. Motorsi, and F. Pollara, "A soft-input softoutput APP module for iterative decoding of concatenated codes," IEEE Communications Letter, vol. 1, pp. 22-24, January 1997.

[17] L. Hanzo, S. X. Ng, T. Keller, and W. Webb, Quadrature amplitude modulation: From basics to adaptive trellis-coded, turbo-equalised and space-time coded OFDM, CDMA and MC-CDMA systems, pp. 746-748. Wiley-IEEE Press, 2nd ed., December 15, 2004.

[18] B. Scanavino, G. Montorsi, and S. Benedetto, "Convergence properties of iterative decoders working at bit and symbol level," in IEEE Global Telecommunications Conference, 2001. GLOBECOM '01., vol. 2, (San Antonio, TX, USA), pp. 1037-1041, 2001.

[19] S. X. Ng, S. Das, J. Wang, and L. Hanzo, "Near-capacity iteratively decoded space-time block coding," in 67th IEEE Vehicular Technology Conference. VTC-'08 Spring., (Marina Bay, Singapore), May 2008. 